\title{
RADIAL VELOCITY VARIATIONS OF THE SECONDARY STAR IN U GEMINORUM
}

\author{
Richard A. Wade \\ Department of Astronomy \\ California Institute of Technology
}

We have used a photon-counting array detector at the coude spectrograph of the $M t$. Wilson $2.5-\mathrm{m}$ reflector to follow changes in the near-infrared spectrum of U Geminorum around most of the orbit. A preliminary analysis of two series of exposures shows radial velocity variations in the $\mathrm{Na} I$ absorption doublet at $\lambda 8183$ and $\lambda 8195$, at the orbital frequency and with the orbital phase appropriate for the M5-type secondary star. A first attempt to detect the $K$ I line at $\lambda 7699$ was unsuccessful.

A tentative value for the radial velocity semi-amplitude $K_{2}$ is $280 \mathrm{~km} \mathrm{sec}{ }^{-1}$. This result is consistent with predicted values of $K_{2}$ (Smak 1976). The indicated mass for the secondary star, about 0.35 solar masses, requires an effective temperature of about $3400 \mathrm{~K}$ if the star is on the main sequence (Veeder 1973). The observed temperature of the secondary star is about $3000 \mathrm{~K}$ (Wade 1979). If the star lies on the main sequence mass-radius line, as it appears to do, then $U$ Gem $B$ is underluminous for its mass and overluminous for its temperature.

\section{REFERENCES}

Smak, J. 1976, Acta Astr., 26, 277. Veeder, G. J. 1973, Thesis, California Inst. of Technology. Wade, R. A. 1979, Astron. J., 82, 562. 